\title{
Markers associated with altitude and ecological factors in hexaploid Campeiostachys nutans (Griseb.) J. L. Yang, B. R. Baum et C. Yen and tetraploid Roegeneria nutans (Keng) Keng
}

\author{
Yu-Xia Guo $\cdot$ Cheng-Zhang Wang • \\ Xue-Bing Yan · Rui-Yong Wang $\cdot$ Sha-Sha Liu • \\ Miao Tian
}

Received: 18 April 2013/Accepted: 8 October 2013/Published online: 20 October 2013

(C) The Author(s) 2013. This article is published with open access at Springerlink.com

\begin{abstract}
The genetic basis of adaptation can be unraveled directly at the genome level, without regard to selectively advantageous genes or traits. Genetic variation and adaptation of polyploidy in the evolution of plants is becoming an area of intense interest. Whether hexaploid wheatgrass species has higher adaptability to altitude than tetraploid or not needs to be proofed by population genomic data. A fluorescence-based AFLP technique was used to investigate the allele share of hexaploid Campeiostachys nutans (Griseb.) J. L. Yang, B. R. Baum et C. Yen and tetraploid Roegeneria nutans (Keng) Keng as well as the genetic variation and natural selection in 22 natural populations and their association with ecological factors. Of all the AFLP bands, $84.29 \%$ were found in common between two different ploidy wheatgrasses. In addition, we found that hexapoid $C$. nutans had 1.45 times the allelic frequency and more large-sized bands than tetraploid $R$. nutans. After genetic analyses, the next factor examined should be the effect of altitude on genetic variation. There were no significant inter-population genetic differentiations, suggesting that distance did not contribute to spatial isolation. Altitude and soil nutrient availability might play an important role in maintaining the genetic diversity. A
\end{abstract}

Y.-X. Guo · C.-Z. Wang · X.-B. Yan (ه) ·

R.-Y. Wang · S.-S. Liu · M. Tian

College of Animal Science and Veterinary Medicine,

Henan Agricultural University, Zhengzhou 450002, China

e-mail: yxbbjzz@163.com certain percentage of positive selection loci were discovered in total genome allele. Hexaploid wheatgrass with higher ploidy numbers can show strong genetic adaptability to adverse high-altitude condition because of its complex genomic background with an additional $\mathrm{H}$ genome, which supports our hypothesis. Though this evolutionary process is believed to be the driving force behind sympatric speciation, we cannot forecast that hexaploid wheatgrass species will diversify into two species in the long term, but believe that diversification under local selection helps to increase the adaptability to the changes in altitude.

Keywords Allelic share - Campeiostachys nutans $\cdot$ Environment $\cdot$ Genetic variation . Polyploid $\cdot$ Roegeneria nutans $\cdot$ Selection

\section{Introduction}

Polyploidization is seen as one of the major processes that has driven and shaped the evolution of higher organisms (Stebbins 1950; Grant 1981). A common assumption is that populations with higher ploidy numbers have higher levels of genetic diversity (Mallet 2007; García-Verdugo et al. 2009), especially if they are allopolyploids and are not of recent origin (Ramsey and Schemske 2002; Luttikhuizen et al. 2007). This assumption has been tested in some population genetic studies that analyzed two (tetraploids and diploids in Luttikhuizen et al. 2007; Abbott et al. 2007) or three 
(García-Verdugo et al. 2009) ploidy levels. Interestingly, opposite findings of lower (Kim et al. 2009) or higher (García-Verdugo et al. 2009) genetic diversity in higher polyploid plants has led to debate about the benefits of polyploidy with regard to genetic diversity. Polyploid evolution independent of the plants' progenitors and habitat preference is a possible explanation for higher diversity and lower polyploidy. In addition to contributing to the genetic diversity of multiploid species, studying polyploidy led to a recent discovery regarding the contribution of polyploidy to the response of species and communities to environmental change (Davis et al. 2005). Genetic variation in a population can be induced and maintained by the force of natural selection in heterogeneous habitats (Zhang 2004). Altitude can also affect the genetic variation and lead to rapid evolution in alpine plant species (Geng et al. 2009). Plant populations rapidly diverge in response to changes in soil conditions (Snaydon and Davies 1982), and a population's genetic variation is related to the level of soil nutrients (Orians et al. 2003). At the same time, soil and eco-environmental conditions have a notable effect on a population's genetic structure along altitudinal gradients (Lazrek et al. 2009). Based on the AFLP molecular marker, genome scans provide a means to identify the genetic basis of such adaptations to adverse environmental conditions such as high altitude without previous knowledge about the particular genetic variants or traits under selection (Fischer et al. 2011).

Allopolyploid Campeiostachys nutans (Griseb.) J. L. Yang, B. R. Baum et C. Yen $(2 n=6 x=42$, StStYYHH) and Roegeneris nutans (Keng) Keng, $(2 n=4 x=28$, StStYY) are very similar in their morphology. The characteristics that best distinguish these two species are chromosome number and genomic constitution (Löve 1984). Evidence from different studies suggest that polyploidy is not an evolutionary dead end (Otto and Whitton 2000), but it does not prove that polyploidization contributes to evolutionary success (In review of Otto 2007). It is often thought that polyploids have an advantage over diploids via their greater ability to mask deleterious mutations. Although the evolutionary success of polyploid plants has been widely recognized, there is virtually no information on how some related polyploid with similar genomes have evolved after their formation. Whether polyploidization increases the fitness and adaptation of a group relative to taxa that have undergone no or less polyploidization is a question that has yet not to be answered. Facing the same selective pressures, whether higher polyploidy Triticeae species may well have undergone beneficial evolutionary transitions? What kind of genetic diversity do different polyploidy Triticeae species made up of $\mathbf{S t}$ and $\mathbf{Y}$ genomes show, and which species shows more genetic variation after examination of a multi-population neutral allele and selection loci by the altitude? No study has examined genetic variation and its association with ecological factors in context with the population genetic evidence of the two Triticeae species. Can these genetic divergences be attributed to the soil change along altitudinal gradients? The answer to these questions will help us to understand the evolutionary history of polyploidy and facilitate the genetic conservation of two important Triticeae species on the QinghaiTibetan Plateau. After we determined the genetic diversity and differentiation at population level form the standpoints of germplasm conservation of two Triticeae species (Yan et al. 2009; Yan et al. 2010), the specific objectives of this investigation are to explore the consanguinity between hexaploid $C$. nutans and tetraploid $R$. nutans, to calculate the genetic relationship at the population level between altitude, soil characters and population genetic indexes, and to test the selection loci under the altitude from the view of genetic basis for the ecological adaptation.

\section{Materials and methods}

Plant materials and soil sampling

Seed samples from 14 populations of $C$. nutans (Griseb.) J. L. Yang, B. R. Baum et C. Yen $(2 n=6 x=42$, StStYYHH) and 8 populations of Roegeneris nutans (Keng) Keng, $(2 n=4 x=28$, StStYY) were collected in Maqin County, Qinghai Province, China. The geographical conditions of the collecting sites are presented in Table 1. Seed samples were collected following the method used in Jin and Lu (2003). Approximately 30 individuals from each population were germinated, and seeds from each spike were planted in a plastic cup that was placed in a greenhouse. After 5-6 weeks, approximately $1 \mathrm{~g}$ of pooled leaf material from 10 seedlings of each spike was harvested, placed in a zip-lock plastic bag, and stored in a freezer $\left(-80^{\circ} \mathrm{C}\right)$ until used. Culture 
Table 1 Geographical condition of sampled populations of two wheatgrasses from the China Qinghai-Tibetan Plateau of

\begin{tabular}{llllll}
\hline Species & Population identity & Altitude $(\mathrm{m})$ & Sample size & Latitude (N) & Longitude (E) \\
\hline C. nutans & E4 & 2,800 & 30 & $34^{\circ} 31^{\prime} 32^{\prime \prime}$ & $100^{\circ} 30^{\prime} 11^{\prime \prime}$ \\
& E5 & 2,940 & 29 & $34^{\circ} 30^{\prime} 56^{\prime \prime}$ & $100^{\circ} 29^{\prime} 18^{\prime \prime}$ \\
& E14 & 3,060 & 22 & $34^{\circ} 31^{\prime} 36^{\prime \prime}$ & $100^{\circ} 29^{\prime} 23^{\prime \prime}$ \\
& E15 & 3,080 & 22 & $34^{\circ} 31^{\prime} 48^{\prime \prime}$ & $100^{\circ} 30^{\prime} 11^{\prime \prime}$ \\
E3 & 3,200 & 28 & $34^{\circ} 31^{\prime} 21^{\prime \prime}$ & $100^{\circ} 28^{\prime} 12^{\prime \prime}$ \\
E13 & 3,260 & 21 & $34^{\circ} 31^{\prime} 1^{\prime \prime}$ & $100^{\circ} 26^{\prime} 47^{\prime \prime}$ \\
E2 & 3,400 & 28 & $34^{\circ} 31^{\prime} 52^{\prime \prime}$ & $100^{\circ} 29^{\prime} 17^{\prime \prime}$ \\
E1 & 3,540 & 26 & $34^{\circ} 31^{\prime} 39^{\prime \prime}$ & $100^{\circ} 27^{\prime} 51^{\prime \prime}$ \\
E11 & 3,680 & 26 & $34^{\circ} 30^{\prime} 57^{\prime \prime}$ & $100^{\circ} 25^{\prime} 56^{\prime \prime}$ \\
E12 & 3,780 & 21 & $34^{\circ} 29^{\prime} 57^{\prime \prime}$ & $100^{\circ} 24^{\prime} 14^{\prime \prime}$ \\
E9 & 3,920 & 27 & $34^{\circ} 30^{\prime} 34^{\prime \prime}$ & $100^{\circ} 24^{\prime} 46^{\prime \prime}$ \\
E6 & 3,940 & 30 & $34^{\circ} 30^{\prime} 47^{\prime \prime}$ & $100^{\circ} 23^{\prime} 58^{\prime \prime}$ \\
E10 & 3,950 & 30 & $34^{\circ} 30^{\prime} 43^{\prime \prime}$ & $100^{\circ} 25^{\prime} 25^{\prime \prime}$ \\
E8 & 4,020 & 19 & $34^{\circ} 30^{\prime} 21^{\prime \prime}$ & $100^{\circ} 23^{\prime} 53^{\prime \prime}$ \\
R5 & 2,940 & 24 & $34^{\circ} 30^{\prime} 56^{\prime \prime}$ & $100^{\circ} 29^{\prime} 18^{\prime \prime}$ \\
R3 & 3,200 & 28 & $34^{\circ} 31^{\prime} 21^{\prime \prime}$ & $100^{\circ} 28^{\prime} 12^{\prime \prime}$ \\
R2 & 3,400 & 29 & $34^{\circ} 31^{\prime} 52^{\prime \prime}$ & $100^{\circ} 29^{\prime} 17^{\prime \prime}$ \\
R1 & 3,540 & 18 & $34^{\circ} 31^{\prime} 39^{\prime \prime}$ & $100^{\circ} 27^{\prime} 51^{\prime \prime}$ \\
R11 & 3,680 & 27 & $34^{\circ} 30^{\prime} 57^{\prime \prime}$ & $100^{\circ} 25^{\prime} 56^{\prime \prime}$ \\
R9 & 3,920 & 28 & $34^{\circ} 30^{\prime} 34^{\prime \prime}$ & $100^{\circ} 24^{\prime} 46^{\prime \prime}$ \\
R6 & 3,940 & 31 & $34^{\circ} 30^{\prime} 47^{\prime \prime}$ & $100^{\circ} 23^{\prime} 58^{\prime \prime}$ \\
R7 & 4,040 & 31 & $34^{\circ} 30^{\prime} 51^{\prime \prime}$ & $100^{\circ} 24^{\prime} 41^{\prime \prime}$ \\
\hline
\end{tabular}

samples were deposited in the Institute of Biodiversity Science, Fudan University in Shanghai. Soil from an underground depth of $0-20 \mathrm{~cm}$ was sampled at 15 sites using a stainless steel soil-sampling probe in August of 2005, and three replicates from each site were taken. After disposing of the pebbles and other wastes, airdried soil samples were placed in zip-lock plastic bags and stored in a freezer $\left(-80^{\circ} \mathrm{C}\right)$ until used.

\section{Fluorescence-based AFLP technique}

A fluorescence-based AFLP (amplified restricted fragment length polymorphisms) technique was used to investigate the genetic variation of 22 natural populations of two wheatgrasses from the Qinghai-Tibetan Plateau at different altitudes. Five selected AFLP primer combinations (E-AAC/M-CAA, E-AAC/M-CTT, E-ACG/MCTT, E-AGG/M-CAG, and E-ACG/M-CTG) were used in this study. A detailed procedure for the fluorescencebased AFLP was described in the publication of Yan et al. (2010), including DNA extraction, digestion, ligation, PCR amplification, electrophoresis and scoring.
Allele frequency inferences based on higher probability of belonging to one ancestral species

We used Structure v.2.2 (downloaded from http:// pritch.bsd.uchicago.edu) and applied the method of examining dominant markers, such as AFLP, with regard to polyploidy, as described in detail by Falush et al. (2007). To explore the allelic share of two Triticeae species with similar genomic constitutions and infer the contribution of the $\mathrm{H}$ genome to genetic differentiation, we adopted the admixture model because it is the most appropriate model for dealing with introgressed populations; it allows for the analysis of admixture and the correlated allelic frequencies (Pritchard et al. 2000a). We used prior information about the populations to assist in clustering and obtained information about the distribution of $Q$ (the estimated membership coefficient) for each individual as well as estimating the mean. Based on a higher $Q$ and a posterior probability, the allelic frequency for each individual was selected for the two wheatgrasses (Pritchard et al. 2000b). 
Principal coordinate analysis of all individuals of two Triticeae species

In order to analyze the genetic relationship of two Triticeae species based on the individual allelic data, the genetic dissimilarities were calculated based on Jaccard's coefficients (1908) between individuals after our former population-level calculation of each species (Yan et al. 2009) using the SIMQUAL option, and they were ordered in a dissimilarity matrix. Genetic dissimilarity (DS) was calculated as DS $=1$ - Jaccard's similarity. Principal coordinate analysis (PCoA) was performed using the DCENTER and EIGEN programs, as described by Gower (1996) in NTSYS v.2.1. This multivariate approach was chosen to complement the cluster analysis because the analysis is more sensitive to closely related individuals, whereas PCoA is more informative regarding distances among major groups (Hauser and Crovello 1982).

\section{Detection of non-neutral loci}

We used the software BayeScan V.2.01 to identify candidate loci under natural selection from genetic data, using differences in allele frequencies between populations based on the multinomial-Dirichlet model following the instruction of Foll and Gaggiotti (2008). Selection is introduced by decomposing $\mathrm{F}_{\text {st }}$ coefficients into a population-specific component (beta) shared by all loci, and a locus-specific component (alpha) shared by all the populations using a logistic regression. Departure from neutrality at a given locus is assumed when the locus-specific component is necessary to explain the observed pattern of diversity (alpha significantly different from 0 ). In this study, three Jeffrey's scales of evidence were defined as "strong selection loci" with $90.9 \%$ confidence corresponding to $\log 10(\mathrm{BF})=1.0(\mathrm{BF}$, Bayesian factor), "very strong selection loci" with $96.9 \%$ confidence corresponding to $\log 10(\mathrm{BF})=1.5$, and "decisive selection loci" with $99 \%$ confidence corresponding to $\log 10(\mathrm{BF})=2.0$. A positive value of alpha suggests diversifying selection, whereas negative values suggest balancing or purifying selection. In our genome scan, we used a threshold for the $\log 10(\mathrm{BF})=1.0$ (strong) for a marker to be considered under selection. This corresponds to a posterior probability greater than $90.9 \%$ for the model accounting for selection.
Correlation analyses between genetic indices and other environmental parameters

The analytical methods and results of soil parameters were described in detail in our publication (Wang et al. 2009). Two methods were used to test the association between genetic indices and other environmental parameters. First, the correlation between the genetic distances and the corresponding geographical origins of the populations, such as altitude, latitude and longitude, were investigated by the Mantel test (Mantel 1967). Second, both the Pearson and Spearman correlation coefficients between the intra-population neutral genetic diversity (calculated on the software Popgen 32 excluding the selection loci) and the other environmental parameters were calculated using the bivariate test in SPSS 11.5 for Windows. All curve regression models were then used to test the correlations between the genetic diversity of the populations and other explanatory factors. The probabilities for the significance of all correlation coefficients were determined followed by a Mann-Whitney $U$ test (adjusting the a-level by Bonferroni inequality). The analysis were performed using the program SPSS.

\section{Results}

Identification of alleles shared by hexaploid $C$. nutans and tetraploid $R$. nutans

Although polymorphisms among the populations of the two species were detected for the majority of fragments, only 210 clear and consistent fragments were used for further genetic evaluation. The overall percentage of polymorphic AFLP loci was $73.52 \%$ for $C$. nutans and $64.13 \%$ for $R$. nutans, suggesting that more alleles are likely to be homozygous within the tetraploid $R$. nutans populations compared to the hexaploid $C$. nutans populations. Bayesian cluster analysis of the two different polyploid wheatgrass species indicated that $84.29 \%$ of all C. nutans and $R$. nutans alleles were consanguineous. The remaining unshared alleles were rarely found in the tetraploid $R$. nutans; however, the shared alleles were commonly detected in hexaploid C. nutans (Fig. 1). The frequency of each allele in hexaploid $C$. nutans was 1.45 times that of tetraploid $R$. nutans, on average. These 
results demonstrate that the tetraploid species $R$. nutans contains only a few rare alleles that are commonly present in the hexaploid species $C$. nutans. Moreover, longer bands (alleles) were more commonly found in hexaploid $C$. nutans for each primer combination (Fig. 2).

Principal coordinate analysis ( $\mathrm{PCoA})$ of all individuals from two wheatgrass species

We examined all individuals from two Triticeae species using principal coordinate analysis (PCoA), which reduces multivariate data sets into trends of maximum relevance. Axes 1 and 2 accounted for 36 and $23 \%$ of the variance, respectively (Fig. 3). Populations of each species formed clusters, but species were not separated by either axes. Populations from similar altitudes and geographical origins did cluster by species. The variance from the tetraploid $R$. nutans individuals was mainly distributed in the first axis, while the variance for the more highly diversified hexaploid $C$. nutans individuals was mainly distributed along both the axes. Tetraploid and hexaploid individuals from the Triticeae species overlap at the approximate center of the two axes.

Selection loci between two polyploidy Triticeae species

Candidate selection loci in two Triticeae species were examined (Fig. 4). Within each species, a certain percentage of selection loci were discovered. Hexaploid $C$. nutans showed a higher percentage of strong

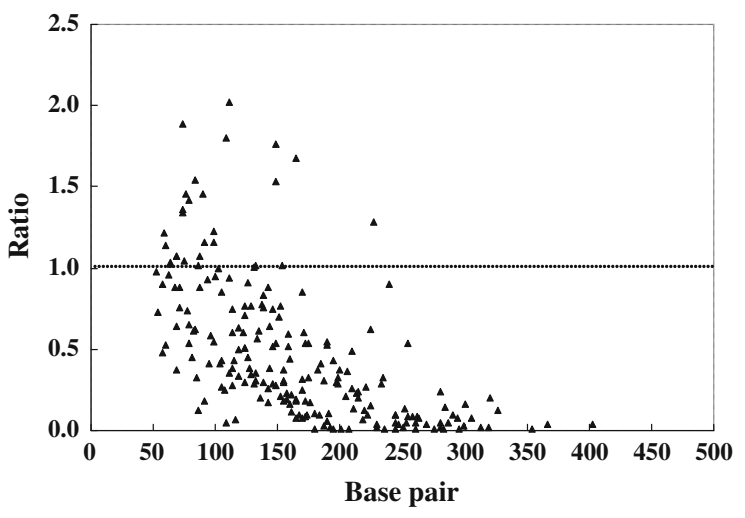

Fig. 2 Relationships between the allele frequency ratio between tetraploid $R$. nutans and hexaploid $C$. nutans and the length of each allele. The $x$-axis represents the length of each allele, while the $y$-axis shows the corresponding allele frequency ratio between $R$. nutans and $C$. nutans

selection loci compared to tetraploid $R$. nutans at three Bayesian factor thresholds (Table 2). A total of 10 loci with the positive Bayesian factor $(\log 10(\mathrm{BF})>0$ ) showed the diversifying selection against two wheatgrass species under the different altitude condition. Most of selection loci showed a larger Fst value under local selection, while minor of selection are expected to show a lower Fst value loci under balancing selection or purifying selection (Fig. 4).

Genetic variation and structure of wheatgrass populations associated with altitude and soil conditions

The sampled $C$. nutans and $R$. nutans populations covered a wide range of altitudes $(1,200 \mathrm{~m})$ and a
Fig. 1 Comparisons of the allele frequency between tetraploid $R$. nutans and hexaploid $C$. nutans. The $x$-axis represents the frequency of an allele in tetraploid $R$. nutans, while the $y$-axis shows the frequency of the same allele in hexaploid $C$. nutans

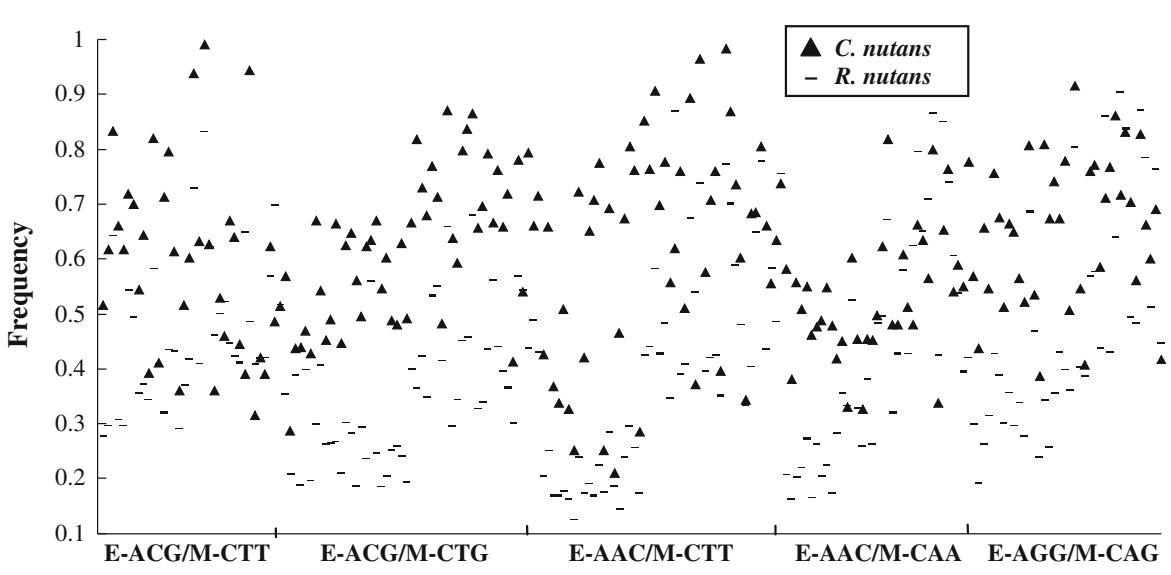




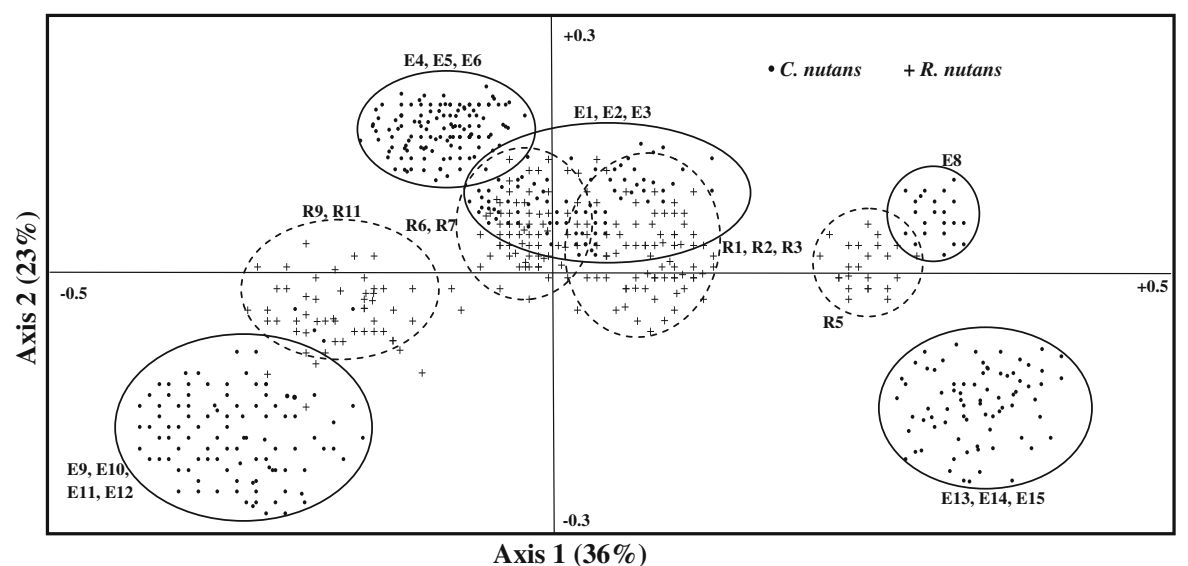

Fig. 3 PCoA analysis of 601 individuals sampled from 22 populations of two Triticeae species. The percentage of the variance explained by each axis is given in parentheses.
Individual samples of $R$. nutans are circled by straight lines and by dotted lines for $C$. nutans

\section{Discussion}

Allelic ancestry and evolutionary comparison between hexaploid $C$. nutans and tetraploid $R$. nutans

In this study, we found a greater number of shared alleles and a high allelic frequency in hexaploid $C$. nutans (StStYYHH) compared to tetraploid $R$. nutans (StStYY). PCoA analysis also indicated a higher genetic variability in hexaploid $C$. nutans than tetraploid $R$. nutans, which is in agreement with our original hypothesis. In the Roegneria genus, the Y genome may have a similar origin and evolutionary history to the St genome (Lu and Salomon 2004; Liu et al. 2006). Different hypotheses regarding the origin of $\mathbf{Y}$ genome have been suggested, and the origin is still under debate (reviewed in Sun and Salomon 2009; Mason-Gamer et al. 2010). Regardless of the origin of $\mathbf{Y}$ genome, it appears that the addition of an $\mathbf{H}$ genome brings complexity to the entire genetic pool of hexaploid $C$. nutans. We deduce that it is the $\mathbf{H}$ genome that leads to differences in the allele frequency and diversity of the two different polyploidy Triticeae species. In a related study, hexaploid bentgrasses (Agrostis gigantea) were found to be genetically distinct from the tetraploid $A$. palustris and have more species-specific markers through the use of AFLPs (Vergara and Bughrara 2004). These findings agree with our hypothesis of higher genetic variability 

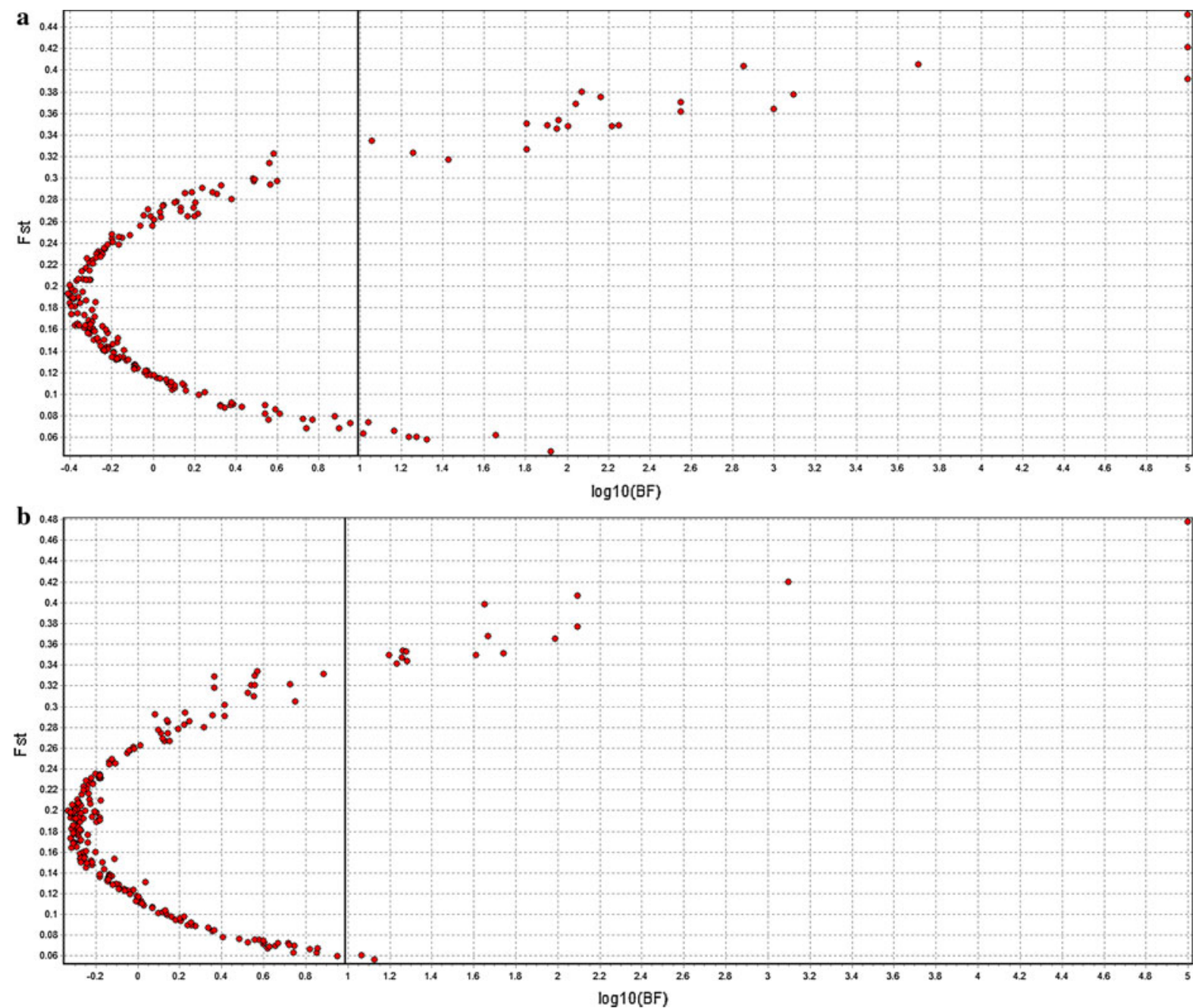

Fig. 4 Bayesian test for selection on individual AFLPs. Approach was implemented in BayeScan for the a Hexaploid $C$. nutans and $\mathbf{b}$ Tetraploid $R$. nutans separately. Fst is plotted against the $\log 10$ of the posterior odds (PO). The vertical line

Table 2 Selection loci and their percentage of two different polyploidy Triticeae species

\begin{tabular}{llll}
\hline Species & $\begin{array}{l}\text { Strong } \\
\text { selection } \\
\text { loci }\end{array}$ & $\begin{array}{l}\text { Very strong } \\
\text { selection loci }\end{array}$ & $\begin{array}{l}\text { Decisive } \\
\text { selection loci }\end{array}$ \\
\hline $\begin{array}{c}\text { Tetraploid } \\
\text { R. nutans }\end{array}$ & $17(8.1 \%)$ & $9(4.3 \%)$ & $4(1.9 \%)$ \\
$\begin{array}{c}\text { Hexaploid } \\
\text { C. nutans }\end{array}$ & $31(14.8 \%)$ & $22(10.5 \%)$ & $15(7.1 \%)$ \\
\hline
\end{tabular}

and allele diversity in hexaploid $C$. nutans than in tetraploid $R$. nutans. A slightly greater allelic diversity per locus and a higher allelic frequency was also shows the critical PO used for identifying outlier markers. The markers on the right side of the vertical line are candidates for being under positive selection

detected in hexaploid Triticum aestivum compared to its tetraploid species ancestor (Alamerew et al. 2004). Although hexaploid $C$. nutans displayed more AFLP variability compared to $R$. nutans, both species are genetically diverse and widely distributed across a diverse range of altitudes on the Qinghai-Tibetan Plateau. C. nutans is the dominant species at high, cold pasture lands (Chen and Jia 2002) and was found to have a higher degree of genetic variability in this study. The hexaploid $C$. nutans species is highly evolved and is indigenous to the high, cold rangeland of the Qinghai-Tibetan Plateau (Wang et al. 2006). Similar situations are also commonly found in other 


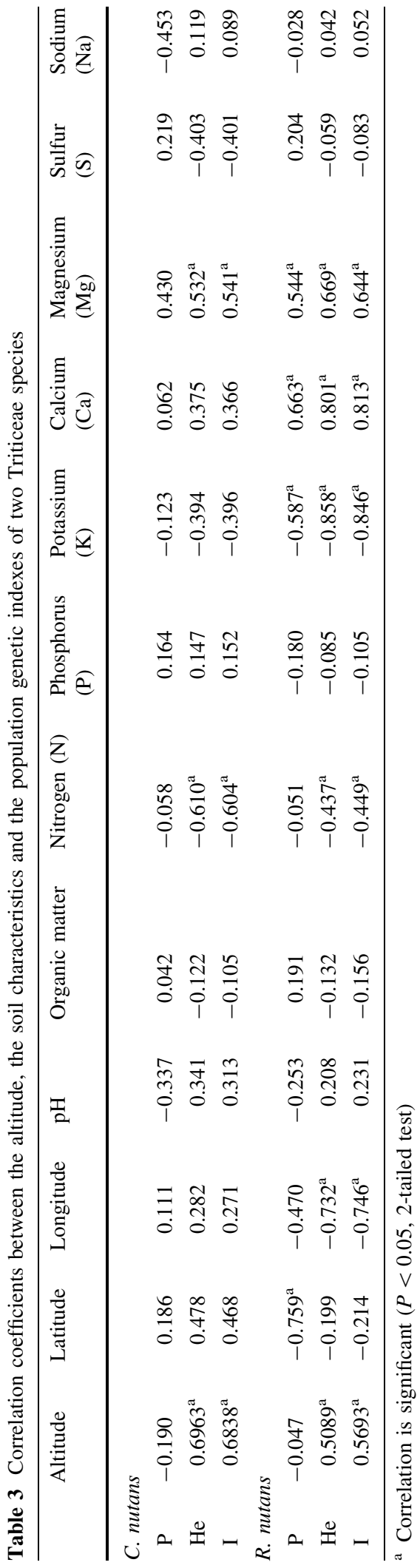

polyploid plants (e.g., Monte et al. 2001; Ohsawa et al. 2007; García-Verdugo et al. 2009). The findings regarding two wheatgrasses indicate that a high degree of polyploidy can provide a strong resistance to adverse conditions, which supports the hypothesis that polyploidy, is a driving force in the evolution of plant species (Stebbins 1950). The Qinghai-Tibetan Plateau represents an important geographical landscape and pasture region in China and is where the Huanghe River, Yangzi River and Lanchang River originate. The hexaploid $C$. nutans species should be studied in greater detail from the perspective of germplasm conservation, ecological importance and the grazing implications on animal husbandry.

Evolutionary process and speciation fate of two polyploidy wheatgrass species

Positive selection acting on a locus increases population divergence, whereas balancing selection keeps it low (Lewontin and Krakauer 1973). Similar forces of selection and evolution experienced by two polyploid species may cause different diversifying and stabilizing selection on the hexaploid and tetraploid wheatgrass species. Loci under local selection are expected to show a larger Fst value than neutral loci, while loci under balancing selection or purifying selection are expected to show a lower Fst value, which means that two wheatgrass species are undergoing more local selection (Fig. 4). Although this evolutionary process is believed to be the driving force behind sympatric speciation, we cannot predict that hexaploid populations will diversify into two species in the long term, but believe that diversification helps to increase the adaptability to the changes in altitude. Based estimates by Otto (2007), populations of hexaploid wheatgrass species may adapt to faster than populations of tetraploid wheatgrass species along altitudinal gradients because mutant alleles are less masked. Selection theory provides two probable mechanisms of heterozygote advantage and frequency-dependent selection by which diversifying selection works to maintain higher population genetic polymorphism of hexaploid species. Hexaploid $C$. nutans individuals may have heterozygote advantage copmared to tetraploid $R$. nutans individuals. In the positive frequency-dependent selection, the fitness of a beneficial phenotype that adapts to the changes in altitude increases as it becomes more common, however in negative frequency-dependent selection, the fitness 
of a recessive phenotype increases as it becomes less common. This finding is another proof that polyploidy wheatgrass species were not an evolutionary dead end (Otto and Whitton 2000), because higher level of polyploidy increases the ratio of marker allele associated with the altitude and the adaptation to the high-cold environment like the Qinghai-Tibetan Plateau and species richness in wild community. Once established, the long-term fate of a polyploidy lineage depends on its ability to adapt. One benefit of a higher ploidy level is that it increases the number of gene copies that can harbor a new beneficial mutation. On the other hand, as Stebbins (1971) argued, polyploidy "dilutes the effects of new mutations" because of masking by nonmutant alleles.

Selection on the genetic variation and structure of wheatgrass populations by altitude and soil conditions

Population structure and history have similar effects on the genetic diversity at all neutral loci. However, some marker loci may also have been strongly influenced by natural selection. If we could identify those loci that have responded to selection during the divergence of populations, then we may obtain better estimates of the parameters of population history by excluding these loci. In this study as well as a close relationship to altitude, the changes in the intrapopulation neutral genetic diversity has a relationship to the soil factors at the different altitudes (such as the available nitrogen, potassium, calcium and magnesium levels), which suggests that the altitude, soil nutrients and climate factors might play an important role in maintaining the neutral genetic diversity of wheatgrass species. Tetraploid $R$. nutans showed more response to selection from soil conditions than the hexaploid species $C$. nutans. This effect is confirmed by a previous study on the genetic diversity of Betula luminifera and its association with ecological factors (Xie et al. 2009).

We detected no correlation between the interpopulation genetic distances and the altitude ranges in two wheatgrass species using the Mantel test, suggesting that there is no isolation due to distance between populations along altitudes, latitudes and longitudes. However, previous studies many $C$. nutans populations significant correlations between the geographical origins, altitude, and genetic distances were observed (Chen et al. 2009; Yan et al. 2007). Differences in the sampling strategies can lead to conflicting conclusions regarding the relationship between the genetic distances and the geographical origins of the samples. Self-fertilization and limited seed dispersal can significantly influence the genetic diversity and differentiation over widely dispersed ( $>60 \mathrm{~km}$ ) Elymus populations (Bockelmann et al. 2003). To explore the vertical changes in population genetics, rather than the horizontal changes, sampled $C$. nutans and $R$. nutans populations from a large a range of altitudes $(1,200 \mathrm{~m})$ within a restricted range of latitudes $(<50 \mathrm{~km}$ horizontally). No genetic isolation by distance between populations along the altitude range must be scientifically demonstrated by notable gene flow among populations. In a previous investigations, we detected a significant gene flow from mediumaltitude wheatgrass populations to low- and highaltitude wheatgrass populations (Yan et al. 2010), which can have a large influence on the linear relationship between the populations' genetic distances and altitude. Some of the above reasons are the basis of the complex genetic differentiations of the two wheatgrass species along altitudinal gradients detected in this study. Apart from the local selection above, no spatial isolation in this study and excess flow among some populations (Yan et al. 2010) have formed the complex genetic structure of two wheatgrass species.

Our findings showed a higher genetic variability in hexaploid $C$. nutans than tetraploid $R$. nutans, which is in agreement with our original hypothesis. Our study demonstrates a considerably correlation between the intra-population genetic diversity and the altitudinal distribution, suggesting that the two wheatgrass species have been under genetic selection by various environments along their altitude gradients. Tetraploid $R$. nutans has more susceptibility to undergo a stronger selection from soil condition and lower population genetic variation than hexaploid $C$. nutans population, Population-level genetic studies of polyploid plants have indicated that polyploidization should no longer be viewed as a rare event producing a polyploid species of unique origin and uniform genotype (Jeffrey 2007). Instead, polyploid species can maintain high levels of segregating genetic variation through the incorporation of genetic diversity from multiple populations of their diploid progenitors. From the above discussion on the allele share, PCoA, simulation of selection lcoi, relationship between genetic indexes 
and environment along with altitude, higher adaptation of hexaploid $C$. nutans with $\mathbf{S t}, \mathbf{Y}$ and $\mathbf{H}$ genome than its concurrent tetraploid $R$. nutans without $\mathbf{H}$ genome is attributed to the population genetic evidence along the altitudinal gradients on the Qinghai-Tibetan Plateau found in this study.

Acknowledgments This study was supported by two grants from the National Foundation Science Commission of China (No. 31372358 and 30901051). We are grateful to Prof. B.R. Lu of Fudan University, Profs. K. Wang, H. Zhou, and Dr. S.Y. Wang from the Institute of Grassland Sciences, China Agricultural University, Dr. Steven R. Larson from Forage \& Range Research Lab, Utah State University for their comments and extensive assistance in the field.

Open Access This article is distributed under the terms of the Creative Commons Attribution License which permits any use, distribution, and reproduction in any medium, provided the original author(s) and the source are credited.

\section{References}

Abbott RJ, Ireland HE, Rogers HJ (2007) Population decline despite high genetic diversity in the new allopolyploid species Senecio cambrensis (Asteraceae). Mol Ecol 16:1023-1033

Alamerew S, Chebotar S, Huang XQ, Roder M, Andreas B (2004) Genetic diversity in Ethiopian hexaploid and tetraploid wheat germplasm assessed by microsatellite markers. Genet Resour Crop Evol 51:559-567

Bockelmann AC, Reusch TBH, Bijsma R, Bakker JP (2003) Habitat differentiation vs. isolation-by-distance: the genetic population structure of Elymus athericus in European salt marshes. Mol Ecol 12:505-515

Chen MJ, Jia ZX (2002) China forage flora. China Agricultural Press, Beijing, pp 119-120

Chen SY, Ma X, Zhang XQ, Chen ZH (2009) Genetic variation and geographical divergence in Elymus nutans Griseb. (Poaceae: Triticeaee) from West China. Biochem Syst Ecol 37:716-722

Davis MB, Shaw RG, Etterson JR (2005) Evolutionary responses to changing climate. Ecology 86:1704-1714

Falush D, Stephens M, Pritchard JK (2007) Inference of population structure using multilocus genotype data: dominant markers and null alleles. Mol Ecol Notes 7:574-578

Fischer MC, Foll M, Excoffier L, Heckel G (2011) Enhanced AFLP genome scans detect local adaptation in high-altitude populations of a small rodent (Microtus arvalis). Mol Ecol 20:1450-1462

Foll M, Gaggiotti OE (2008) A genome scan method to identify selected loci appropriate for both dominant and codominant markers: a Bayesian perspective. Genetics 180:977-993

García-Verdugo C, Fay MF, Granado-Yela C, De Casas RR, Balaguer L, Besnard G, Vargas P (2009) Genetic diversity and differentiation processes in the ploidy series of Olea europaea L.: a multiscale approach from subspecies to insular populations. Mol Ecol 18:454-467

Geng YP, Tang SQ, Tashi T, Song ZP, Zhang GG, Zeng LY, Zhao JY, Wang L, Shi J, Chen JK, Zhong Y (2009) Fineand landscape-scale spatial genetic structure of cushion rockjasmine, Androsace tapete (Primulaceae), across southern Qinghai-Tibetan Plateau. Genetica 135:419-427

Gower JC (1996) Some properties of latent root and vector methods used in multivariate analysis. Biometrika 53: 325-338

Grant V (1981) Plant speciation. Columbia University Press, New York

Hauser LA, Crovello TJ (1982) Numerical analysis of genetic relationships in Thelypodieae (Brassicaceae). Syst Bot 7:249-268

Jaccard P (1908) Nouvelles recherches sur la distribution florale. Bull Soc Vaud Sci Nat 44:223-270

Jeffrey ZC (2007) Genetic and epigenetic mechanisms for gene expression and phenotypic variation in plant polyploids. Annu Rev Plant Biol 58:377-406

Jin Y, Lu BR (2003) Sampling strategy for genetic diversity. Biodivers Sci 11:155-161

Kim C, Shin H, Choi HK (2009) Genetic diversity and population structure of diploid and polyploid species of Isoëtes in east Asia based on amplified fragment length polymorphism markers. Int J Plant Sci 170:496-504

Lazrek F, Roussel V, Ronfort J, Cardinet G, Chardon F, Aouani ME, Huguet T (2009) The use of neutral and non-neutral SSRs to analyse the genetic structure of a Tunisian collection of Medicago truncatula lines and to reveal associations with eco-environmental variables. Genetica 135:391-402

Lewontin RC, Krakauer J (1973) Distribution of gene frequency as a test of the theory of the selective neutrality of polymorphisms. Genetics 74:175-195

Liu Q, Ge S, Tang H, Zhang X, Zhu G, Lu BR (2006) Phylogenetic relationships in Elymus (Poaceae: Triticeaee) based on the nuclear ribosomal internal transcribed spacer and chloroplast trnL-F sequences. New Phytol 170:411-420

Löve À (1984) Conspectus of the Triticeaee. Feddes Repert 95(7-8):425-521

Lu BR, Salomon B (2004) Differentiation of the StY genomes in Elymus species as referred by meiotic pairing in interspecific hybrids and its evolutionary significance. Chin Biodivers 12:213-226

Luttikhuizen PC, Stift M, Kuperus P, van Tienderen PH (2007) Genetic diversity in diploid vs. tetraploid Rorippa amphibian (Brassicaceae). Mol Ecol 16:3544-3553

Mallet J (2007) Hybrid speciation. Nature 446:279-283

Mantel N (1967) The detection of disease clustering and a generalized regression approach. Cancer Res 27:209-220

Mason-Gamer RJ, Burns MM, Naum M (2010) Phylogenetic relationships and reticulation among Asian Elymus (Poaceae) allotetraploids: analyses of three nuclear gene trees. Mol Phylogenet Evol 54:10-22

Monte JV, De Nova PJG, Soler C (2001) AFLP-based analysis to study genetic variability and relationships in the Spanish species of the genus Aegilops. Hereditas 135:233-238

Ohsawa T, Tsuda Y, Saito Y, Sawada H, Ide Y (2007) Altitudinal genetic diversity and differentiation of Quercus crispula in the Chichibu Mountains, central Japan. Int J Plant Sci 168:333-340 
Orians CM, Lower S, Fritz RS, Roche BM (2003) The effects of plant genetic variation and soil nutrients on secondary chemistry and growth in a shrubby willow, Salix sericea: patterns and constraints on the evolution of resistance traits. Biochem Syst Ecol 31:233-247

Otto SP (2007) The evolutionary consequences of polyploidy. Cell 131:452-462

Otto SP, Whitton J (2000) Polyploid incidence and evolution. Annu Rev Genet 34:401-437

Pritchard JK, Stephens M, Donnelly P (2000a) Inference of population structure using multilocus genotype data. Genetics 155:945-959

Pritchard JK, Stephens M, Rosenberg NA, Donnelly P (2000b) Association mapping in structured populations. Am J Hum Genet 67:170-181

Ramsey J, Schemske DW (2002) Neopolyploidy in flowering plants. Ann Rev Ecol Evol Syst 33:589-639

Snaydon RW, Davies TM (1982) Rapid divergence of plant populations in response to recent changes in soil conditions. Evolution 36:289-297

Stebbins GL (1950) Variation and evolution in plants. Columbia University Press, New York

Stebbins GL (1971) Processes of organic evolution, 2nd edn. Prentice-Hall, Englewood Cliffs

Sun GL, Salomon B (2009) Molecular evolution and origin of tetraploid Elymus species. Breed Sci 59:487-491

Vergara GV, Bughrara SS (2004) Genetic differentiation of tetraploid creeping bentgrass and hexaploid redtop bentgrass genotypes by AFLP and their use in turfgrass breeding. Crop Sci 44:884-890

Wang WY, Wang QJ, Li SX, Wang G (2006) Distribution and species diversity of plant communities along transect on the northeastern Tibetan Plateau. Biodivers Conserv 15: $1811-1828$

Wang RY, Liu SS, Wang CZ, Guo YX, Yan XB (2009) Analysis on soil physical and chemical indices at different altitutdes in alpine rangeland. Acta Agrestia Sin 17:621-628

Xie YQ, Li ZZ, Huang RZ, Xiao XX, Huang Y (2009) Genetic diversity of Betula luminifera populations at different elevations in Wuyi Mountain and its association with ecological factors. Front For China 4:90-95

Yan XB, Guo YX, Zhou H, Wang K (2007) Effect of geographical factors on genetic variation of Elymus nutans Indigenous in the Qinghai-Tibetan Plateau. Acta Bot Boreal Occident Sin 27:0328-0333

Yan XB, Guo YX, Zhao C, Liu FY, Lu BR (2009) Intra-population genetic diversity of two wheatgrass species along altitude gradient on the Qinghai-Tibetan Plateau: its implication for conservation and utilization. Conserv Genet 10:359-367

Yan XB, Guo YX, Zhang C, Liu FY, Lu BR (2010) Population structure affected by excess gene flow in self-pollinating Elymus nutans and E. burchan-buddae (Triticeaee: Poaceae). Popul Ecol 52:233-241

Zhang DY (2004) Plant life history evolution and reproductive ecology. Science press, Beijing 